\title{
Adaptive Wavelet Transforms for Image Coding using Lifting
}

\section{Roger Claypoole Geoffrey Davis Wim Sweldens Richard Baraniuk*}

Image compression relies on efficient representations of images, and within smooth image regions, the wavelet transform provides such a representation. However, near edges, wavelet coefficients decay slowly and are expensive to code. We focus on improving the transform by incorporating adaptivity. Construction of non-linear filter banks has been discussed $[1,2]$, but the question of how to utilize the non-linearities remained. We answer this question by describing our transform via lifting [3].

Lifting provides a spatial domain framework for the wavelet transform. In the lifting formalism, wavelet coefficients are seen as prediction residuals from a linear prediction operation. Wavelet coefficients are large near edges because the linear predictors are built to interpolate low order polynomials. Our goal is to avoid this problem by adapting the predictor based on local image properties. In smooth regions of the image, we use high order polynomial predictors. We adaptively reduce the prediction order to avoid attempting to predict values across discontinuities.

The adaption mechanism complicates our transform and makes it non-linear. There are some important issues we must resolve. First, how do we interpret the scaling function coefficients in our non-linear transform? Second, how do we ensure that the new non-linear transform will be stably invertible?

We address both issues by building our non-linear transform from an unconventional form of a linear wavelet transform [4]. Our starting point is a wavelet transform that factors into an updating lifting step followed by a predicting lifting step (more standard transforms perform these steps in the opposite order). This modification enables us to construct a stably invertible transform and ensures that we can maintain synchronization between the encoder and the decoder.

For our adaptive transform we choose an $N \in\{1,3,5,7\}$ predictor by analyzing each 7-point prediction window. Because the decoder and encoder are synchronized, they make identical prediction decisions. For a given bit rate, our adaptive transform results in sharper horizontal and vertical edges with reduced ringing. However, there are still some ringing artifacts along diagonals, since we use a separable transform; future work will address this issue by utilizing non-separable prediction windows.

[1] F. J. Hampson and J. C. Pesquet. A nonlinear subband decomposition with perfect reconstruction. In Proc. ICASSP, 1996.

[2] R. de Quieroz, D. A. F. Florêncio, and R. W. Schafer. Non-expansive pyramid for image coding using a non-linear filter bank. IEEE Trans. Image Processing, 1996.

[3] W. Sweldens. The lifting scheme: A custom-design construction of biorthogonal wavelets. Journal of Appl. and Comput. Harmonic Analysis, 3(2):186-200, 1996.

[4] R. Claypoole, G. Davis, W. Sweldens, and R. Baraniuk. Nonlinear Wavelet Transforms for Image Coding. Proc. 31st Asilomar Conf., 1997

${ }^{*}$ R. Claypoole and R. Baraniuk are with Rice University; G. Davis is at Dartmouth College; W. Sweldens is with Bell Laboratories, Lucent Technologies. This work supported by an NSF Mathematical Sciences Postdoctoral Research Fellowship, NSF grant MIP-9457438, and ONR grant N00014-95-1-0849. Email: clayporl@rice.edu, gdavis@cs.dartmouth.edu, wim@bell-labs.com, richb@rice.edu. 